\title{
Phenolic Inhibitors of Saccharification and Fermentation in Lignocellulo-Starch Prehydrolysates and Comparative Efficacy of Detoxification Treatments
}

\author{
Madhanamohanan G. Mithra and Gourikutty Padmaja \\ Division of Crop Utilization, ICAR-Central Tuber Crops Research Institute \\ Thiruvananthapuram-695 017, Kerala, India \\ padmajabn@yahoo.com
}

\begin{abstract}
The generation of phenolic inhibitors in the prehydrolysates from steam, dilute sulphuric acid (DSA) and lime pretreated root wastes (peels from sweet potato, elephant foot yam, tannia, beet root and greater yam) and vegetable processing residues (peels from ash gourd, pumpkin and vegetable banana and mixed vegetable waste) was monitored. Further, the effect of detoxification agents such as Tween 20, PEG 4000, active charcoal and sodium borohydride in reducing their levels was compared. The aqueous extracts from native biomass contained soluble phenolics at levels of $1.03 \%$ to $3.02 \%$. Highest release (12.5-16.2\% increase from the values in aqueous extracts of native biomass) of total soluble phenolics (TSPs) into the prehydrolysates was found in steam (60 min.) and DSA (60 min; $\left.121{ }^{\circ} \mathrm{C}\right)$ pretreatments, irrespective of the type of biomass, while lime pretreatment resulted in only 1.7$3.1 \%$ increase in the phenolics. Maximum quantity of TSPs was removed in $2 \mathrm{~h}$ at room temperature $\left(30 \pm 1{ }^{\circ} \mathrm{C}\right)$ by Tween 20 (70-81\%) or its combination with PEG (73-82\%). Active charcoal $\left(2 \% ; 45{ }^{\circ} \mathrm{C} ; 1 \mathrm{~h}\right)$ or sodium borohydride (40 mM; 20 min; $30 \pm 1{ }^{\circ} \mathrm{C}$ ) removed only $40-59 \%$ and $37-53 \%$ phenolics respectively. Loss of reducing sugars was the least (2-3\%) in Tween $20(0.50 \%)$ treatment, indicating its superiority over the other treatments.
\end{abstract}

Keywords: Lignocellulo-starch prehydrolysates, Phenolic inhibitors, Detoxification, Surfactants, Charcoal, Borohydride, Reducing sugar loss.

(C) Copyright 2016 Authors - This is an Open Access article published under the Creative Commons Attribution License terms (http://creativecommons.org/licenses/by/3.0). Unrestricted use, distribution, and reproduction in any medium are permitted, provided the original work is properly cited.

\section{Introduction}

Lignocellulosic biomass has been globally recognized as the most attractive source for bioethanol production, owing to the cheap and abundant availability. Nevertheless, the sustainable production depends on the successful overcoming of biomass recalcitrance and other technological barriers such as high enzyme costs, low conversion rate, generation of saccharification/fermentation inhibitors etc. [1-3]. Pretreatment of biomass is the key step to breaking biomass recalcitrance and enhancing the accessibility of cellulose to enzymatic hydrolysis [3]. Besides disrupting the crystalline structure of cellulose, pretreatment also helps the lignin shield to be broken down and part of the hemicelluloses to be solubilised $[4,5]$. Pretreatment operations generally adopted include steam, acid or alkaline exposures at high temperatures [4] and result in a solid fraction enriched with amorphous cellulose that is easily susceptible to enzymatic hydrolysis along with partially degraded lignin and a soluble prehydrolysate rich in monomeric sugars released from hemicellulose along with lignin degradation products [6]. Most of the lignocellulose derived by-products are inhibitory to fermentation organisms and based on the origin, they have been grouped into weak acids, furan derivatives and phenolic compounds [7, 8]. The extent of formation of these inhibitors depends on the composition of biomass, nature and severity of pretreatment conditions etc. [9]. Phenolic inhibitors are mainly formed during pretreatment from the degradation of lignin and to a 
small extent from the extractives as well as the phenolic ester groups of hemicellulose and include hydroxycinnamic acid, vanillin, syringaldehyde, tannic acid, gallic acid etc. [10-12]. Tejirian and Fu [13] reported the inhibition of cellulolytic enzymes by simple and oligomeric phenols generated during pretreatment from lignin modification/degradation. Phenolic compounds in the soluble fraction have been reported to inhibit microbial growth by possibly interfering with the cell membrane $[14,15]$. Clarke and Mackie [16] found that lower molecular weight phenols had the strongest toxicity to yeast growth. Detoxification of prehydrolysates has been attempted by several researchers using chemical, physical or biological methods [17-19]. Surfactants such as Tween and polyethylene glycol have been reported to reduce the levels of lignin degradation products and enhance the saccharification rate $[13,20,21]$. Adsorption on active charcoal has been attempted by others to reduce the toxicity of hydrolysates [22, 23]. In situ detoxification with sodium borohydride has been reported to tremendously improve the fermentation of sugarcane bagasse hydrolysate [24].

Although lignocellulosic biomasses generally comprise of agricultural residues, woody forest biomass and dedicated grasses such as switchgrass or Miscanthus sp., a major contribution is currently from processing residues also due to increased industrial and domestic activities. We had earlier reported the importance of root and vegetable processing residues as promising feedstocks for bioethanol production, capable of reducing environmental pollution from their inadequate disposal strategies [25-27]. As different from the typical lignocellulosic biomasses (LCBs), these residues also contain appreciable quantities of starch, enabling them to be grouped as lignocellulo-starch wastes. Pretreatment techniques such as steam, dilute sulphuric acid (DSA) and lime (calcium hydroxide) treatments were found to deconstruct the cellulose by partial solubilisation of lignin and moderate to high hydrolysis of hemicellulose and starch. It was reported earlier that steam pretreatment brought about $\mathrm{Ca}$. 53\% and $25 \%$ hydrolysis of hemicelluloses and starch, while DSA hydrolyzed $83-94 \%$ starch and $42-48 \%$ hemicelluloses, with $27-29 \%$ delignification in the former [25]. On the contrary, lime pretreatment solubilised hemicellulose to a limited extent ( $\mathrm{Ca} .12 \%)$, with minimal depolymerisation of starch [26]. Being rich in fermentable sugars, the liquid phase or prehydrolysates from the processing residues of roots (sweet potato, elephant foot yam, tannia, beet root and greater yam) and vegetables (ash gourd, pumpkin, vegetable banana and mixed vegetable wastes) is also a valuable feedstock and combined or whole slurry fermentation has to be considered in these cases. Hence, the monitoring of build-up of fermentation inhibitors and their effective removal from the prehydrolysates become obligatory to enhance the fermentable sugar yield at the saccharification stage. In the present study, the generation of phenolic inhibitors in steam, DSA and lime pretreated root and vegetable processing residues was studied. Besides, the comparative efficacy of various detoxification approaches including surfactant and sodium borohydride application, active charcoal adsorption etc. in reducing their levels in the prehydrolysates was also investigated.

\section{Materials and Methods}

\subsection{Raw Materials}

Peels from five root crops such as sweet potato (Ipomoea batatas), elephant foot yam (Amorphophallus paeoniifolius), tannia (Xanthosoma sagittifolium), beet root (Beta vulgaris) and greater yam (Dioscorea alata) and three vegetables such as ash gourd (Benincasa hispida), pumpkin (Cucurbita moschata) and vegetable (cooking) banana (Musa spp. ABB) were selected for the study. Besides, mixed vegetable waste (comprising peels, seeds, non-edible pulp, rotten vegetables etc.) collected from households and local restaurants was also subjected to the study. Samples were dried and powdered to particle size of $C a$. 2-3mm, as described earlier [25, 27]. The unscreened powders were subjected to steam, dilute sulphuric acid (DSA) and lime pretreatments.

\subsection{Pretreatments}

The most effective pretreatments with regard to hemicellulose and starch hydrolysis coupled with lignin degradation from earlier studies [25-27] were selected for the present study. In simple steam treatment (ST), the dry biomass powders (5.0 g) were moistened to $40 \%$ moisture content with water and then exposed to steam in a Vegetable Steamer (M/s TTK Prestige India Ltd., India) for $45 \mathrm{~min}$. and $60 \mathrm{~min}$. After steam exposure, volume was made up to $50 \mathrm{ml}$ with distilled water, mixed well and centrifuged at $3000 \mathrm{rpm}$ for 20 min. The clear supernatant was stored at $4{ }^{\circ} \mathrm{C}$ till use. In the DSA treatment, $5.0 \mathrm{~g}$ biomass powders were 
suspended in $50 \mathrm{ml}$ DSA $(0.5 \% \mathrm{v} / \mathrm{v})$ and treated at 121 ${ }^{0} \mathrm{C}$ and $0.102 \mathrm{MPa}$ pressure in a Pressure cooker $(\mathrm{M} / \mathrm{s}$ TTK Prestige India Ltd., India) for $60 \mathrm{~min}$. (time after pressure build-up). Samples after cooling were treated as described before. Dry biomass residues (10\% w/v) were also subjected to lime pretreatment at room temperature $\left(30 \pm 1{ }^{\circ} \mathrm{C}\right)$ for $24 \mathrm{~h}$ and $48 \mathrm{~h}$ and at high temperature $\left(121{ }^{0} \mathrm{C}\right.$; $\left.0.102 \mathrm{MPa}\right)$ for $60 \mathrm{~min}$., using calcium hydroxide $(0.1 \mathrm{~g} / \mathrm{g}$ biomass $)$ as described elsewhere [26]. The clear supernatants from the various treatments (hereinafter referred to as prehydrolysates) were used for the detoxification experiments. In the case of all the prehydrolysates and the aqueous extracts from native biomass, the $\mathrm{pH}$ was adjusted to 6.0 and volume increased to the nearest before subjecting to the detoxification treatments.

\subsection{Detoxification Treatments}

Two non-ionic surfactants, i.e., Tween 20 [Poly (oxyethylene) 20 $_{20}$ sorbitan-monolaurate] and PEG 4000 [poly (ethylene glycol) 4000] as well as active charcoal and sodium borohydride or their combinations were supplemented to the prehydrolysates and their comparative efficacy in removing the soluble phenolics was investigated. Tween $20(0.50 \%$ or $0.75 \% \mathrm{v} / \mathrm{v})$ and PEG $(0.25 \%$ or $0.50 \% \mathrm{w} / \mathrm{v})$ were added to separate lot of prehydrolysates and incubated at room temperature for $2 \mathrm{~h}$ with occasional shaking. Immediately after $2 \mathrm{~h}$, the total soluble phenolics (TSP) content in each of the surfactant added sample was assayed. In the combination treatment, the level of Tween 20 was reduced to $0.25 \%(\mathrm{v} / \mathrm{v})$, while retaining the concentration of PEG as $0.25 \%(\mathrm{w} / \mathrm{v})$.

Four types of detoxification treatments were attempted using active charcoal such as $1 \%$ and $2 \%$ $(\mathrm{w} / \mathrm{v})$ levels at room temperature and at $45{ }^{\circ} \mathrm{C}$ for $1 \mathrm{~h}$ each, after which the clear supernatants obtained through centrifugation were used for quantification of phenols in the prehydrolysates and native extracts. Sodium borohydride was also added at two levels such as $20 \mathrm{mM}$ and $40 \mathrm{mM}$ with occasional shaking at room temperature for $20 \mathrm{~min}$. and the TSPs were quantified. In the combination treatments, active charcoal $(1 \%$ $\mathrm{w} / \mathrm{v}$ ) and sodium borohydride (20 $\mathrm{mM})$ were added and kept for $1 \mathrm{~h}$ at room temperature and the TSPs were assayed. A parallel set of native extracts was also kept in all cases. The levels of the detoxification chemicals were finalised based on the earlier studies using Tween 20 from our laboratory as well as from other research reports [20, 22, 24, 28].

\subsection{Total Soluble Phenolics in the Prehydrolysates}

Total soluble phenolics (TSPs) were determined in the non-detoxified and detoxified prehydrolysates using Folin-Ciocalteu reagent [29] and expressed as gallic acid equivalents $(\mathrm{g} / \mathrm{L})$ computed using pure gallic acid standard (SIGMA). Original (native) biomass samples were also extracted with distilled water in the same solid: liquid ratio $(1: 10 \mathrm{w} / \mathrm{v})$ at room temperature $\left(30 \pm 1{ }^{\circ} \mathrm{C}\right)$, with occasional stirring and the TSPs were determined by the same method. Any interference from the surfactants or the other detoxification agents in the assay was nullified by keeping a blank containing the same concentration of detoxification chemicals as in the test samples.

\subsection{Reducing Sugar Loss}

The loss of reducing sugars (RS) brought about by each of the detoxification agents was studied by assaying the RS in the sample extracts prior to and after the detoxification treatment by the Nelson Somogyi method [30]. Interference from the detoxification agents was nullified using blank systems containing the same concentration of the detoxification chemicals for each sample.

\subsection{Statistical Analysis}

Three replicates were kept for each experiment and duplicate analyses were performed on each replicate. The data were subjected to Analysis of Variance (ANOVA) for statistical testing of the mean values and least significant difference (LSD) for pairwise comparison of mean values was found out using the statistical package, SAS 9.3 [31] Each value is considered significant at $\mathrm{p}<0.05$.

\section{Results and Discussion}

\subsection{Generation of Soluble Phenolics During Pretreatment}

The concentration of soluble phenolic compounds in the prehydrolysates from root and vegetable processing residues subjected to steam, DSA and lime treatments was monitored and compared with that released by aqueous extraction from native (untreated) biomass. The original biomass extracts were found to contain high levels of soluble phenolics and the total concentration ranged from 1.03-3.02 g/L (Table 1). Least concentration was found in the aqueous extracts from beet root peel, while very high concentration of 2.9-3.02 g/L was found in ash gourd, pumpkin and 
vegetable banana peels. Phenolics in higher plants range from low molecular weight compounds to polymeric lignin type compounds and these have definite defensive role in the plants, accumulating in higher levels in peels than in edible parts [32]. Many of the simple phenolics have high solubility in water and could therefore exert appreciable inhibition on cellulases unless otherwise they are effectively removed [33].

Pretreatments such as steam (45 and $60 \mathrm{~min}$.), DSA (60 min.) and lime [24 and $48 \mathrm{~h}$ at room temperature $\left(30 \pm 1{ }^{\circ} \mathrm{C}\right)$ and $60 \mathrm{~min}$. at $\left.121^{\circ} \mathrm{C}\right]$ resulted in the release of phenols into the liquid phase in varying amounts. Highest release was observed in the case of steam (60 min.) and DSA (60 min.) and the percentage increase ranged from $12.5 \%$ to $16.2 \%$ in the different biomasses (Table 1). Lime treatment led to an increase of only $1.7 \%$ to $3.1 \%$ which was negligible. During pretreatment, native lignin is demethylated, solubilised (resulting in the release of monomeric and oligomeric phenols) or undergoes degradation changes [34, 35]. Although insoluble lignin is reported to remain in the solid fraction, many simple and oligomeric phenols move into the liquid fraction $[15,36]$. The low molecular weight phenols have a stronger inhibitory effect on fermentative fungi or bacteria and also have deactivating effect on cellulases and $\beta$-glucosidases [1113]. Soluble phenolic levels of $1.3 \mathrm{~g} / \mathrm{L}$ and $2.0 \mathrm{~g} / \mathrm{L}$ have been reported in pretreated maple pinchip liquid [37] and Eucalyptus globulus prehydrolysate [38] respectively, which was lower than that obtained in the present study. Jönsson et al [39] found that soluble phenolics such as 4-hydroxyl benzoic acid, vanillin and catechol were present in the untreated willow hemicellulose extracts, which corroborated with our findings that the native aqueous extracts could also exert potential toxicity to cellulases, by virtue of its high content of soluble phenolics. Besides lignin degradation, phenolic ester groups of hemicellulose are also reported to be sources of soluble phenolics in prehydrolysates [10]. Although dilute acid pretreatment led to solubilisation of hemicelluloses and fracture of lignin, much of the lignin was reported to remain in the residue itself [40] and we also found that only $12.5-14.4 \%$ increase occurred in soluble phenols in the prehydrolysates from the DSA pretreated biomasses under study. Similarly, formation of lignin-Ca-lignin linkages in lime pretreated residues has been reported preventing the release of lignin fragments into the soluble fraction [41]. The small increase in phenols in the lime pretreated root and vegetable residues might be due to such cross linking with lignin.

\subsection{Detoxification of Prehydrolysates}

In the case of most of the lignocellulosic feedstocks, subjected to pretreatments other than acid, the solid fraction is separated from the liquid and subjected to enzymatic saccharification. Removal of the bulk of the liquid phase could also eliminate the inhibition from lignin and carbohydrate degradation products. However, in the case of the root and vegetable processing residues, most of the starch also gets hydrolyzed/depolymerised to lower molecular weight fractions along with hemicelluloses solubilisation/hydrolysis and hence removal of the prehydrolysate could amount to considerable loss of fermentable sugars. Detoxification of prehydrolysate is thus obligatory in this case to improve saccharification and fermentation performance. Some of the detoxification methods such as use of non-ionic surfactants (Tween 20 and PEG 4000), active charcoal and sodium borohydride either alone or in combination were tried in the present study to understand their comparative efficacy in channelling out the soluble phenolics from prehydrolysates.

\subsubsection{Non-ionic surfactants}

Irrespective of the type of biomass or pretreatment techniques, $70-80 \%$ phenols were removed by Tween 20 (0.50\%). Although slightly higher percentage removal of phenols was observed with $0.75 \%$ Tween 20 , the effect could not justify the use of higher levels compared to $0.50 \%$ (Tables 2 a and b). Poly (ethylene glycol) (PEG 4000) exerted much less effect and the percentage removal ranged from $48-65 \%$ in the various pretreated biomass hydrolysates. Total soluble phenolics (TSPs) were removed to the highest extent by both Tween 20 and PEG from the prehydrolysates of steam treated $(60 \mathrm{~min}$.) residues (Table $2 \mathrm{a}$ and $\mathrm{b}$ ), which also had the highest level of TSPs in the prehydrolysates. Kim et al [37] found that the most inhibitory compound formed in lignocellulosic hydrolysates from hot water, steam explosion and dilute acid treatments were phenolics which reduced the cellulose hydrolysis by $50 \%$ and they could recover the full cellulase activity by removing the phenols. Water soluble phenolic acids such as tannic acid and gallic acid were reported to inhibit and deactivate $\beta$ glucosidase enzymes from Trichoderma reesei and Aspergillus niger [11, 12]. These studies stressed the 
need to remove soluble phenolics from the prehydrolysates which could also help reduce the enzyme loading. Phenolic compounds were also reported to exert toxicity to the fermenting organisms by partitioning into biological membranes and causing loss of integrity [42]. Hardwood hydrolysates rich in 4hydroxybenzoic acid were inhibitory to the growth of
Saccharomyces cerevisiae at 1g/L [43]. Enhanced enzymatic conversion of lignocellulose has been reported by several researchers, by supplementing with non-ionic surfactants such as Tween or PEG $[20,21]$ and the major effect reported was the prevention of adsorption of cellulases to exposed lignin surfaces.

Table 1. Total soluble phenolic (TSP) inhibitors in the prehydrolysates $(\mathrm{g} / \mathrm{L}$ ) from root and vegetable processing residues subjected to various pretreatments.

\begin{tabular}{|c|c|c|c|c|c|c|c|}
\hline Biomass residues & Native* & $\begin{array}{l}\text { ST } \\
45 \mathrm{~min} .\end{array}$ & $\begin{array}{l}S T \\
60 \mathrm{~min} .\end{array}$ & $\begin{array}{l}\text { DSA } \\
60 \mathrm{~min} .\end{array}$ & $\begin{array}{l}\text { LRT } \\
24 \mathrm{~h}\end{array}$ & $\begin{array}{l}\text { LRT } \\
48 \mathrm{~h}\end{array}$ & $\begin{array}{l}\text { LHT } \\
60 \mathrm{~min} .\end{array}$ \\
\hline \multicolumn{8}{|c|}{ Root crop processing residues } \\
\hline Sweet potato peel & $2.746^{c}$ & $\begin{array}{l}2.864^{\mathrm{b}} \\
(4.30)^{* *}\end{array}$ & $\begin{array}{l}3.142^{\mathrm{a}} \\
(14.41)\end{array}$ & $\begin{array}{l}3.141^{\mathrm{a}} \\
(14.40)\end{array}$ & $\begin{array}{l}2.825^{\mathrm{b}} \\
(2.88)\end{array}$ & $\begin{array}{l}2.802^{\mathrm{bc}} \\
(2.03)\end{array}$ & $\begin{array}{l}2.813^{b} \\
(2.42)\end{array}$ \\
\hline $\begin{array}{l}\text { Elephant foot yam } \\
\text { peel }\end{array}$ & $2.548^{\mathrm{c}}$ & $\begin{array}{l}2.661^{\mathrm{b}} \\
(4.44)\end{array}$ & $\begin{array}{l}2.946^{\mathrm{a}} \\
(15.63)\end{array}$ & $\begin{array}{l}2.902^{a} \\
(13.88)\end{array}$ & $\begin{array}{l}2.625^{b} \\
(3.02)\end{array}$ & $\begin{array}{l}2.598^{c} \\
(1.95)\end{array}$ & $\begin{array}{l}2.601^{b c} \\
(2.09)\end{array}$ \\
\hline Tannia peel & $2.780^{c}$ & $\begin{array}{l}2.879 \mathrm{bc} \\
(3.56)\end{array}$ & $\begin{array}{l}3.224^{\mathrm{a}} \\
(15.94)\end{array}$ & $\begin{array}{l}3.175^{\mathrm{b}} \\
(14.18)\end{array}$ & $\begin{array}{l}2.855^{b c} \\
(2.67)\end{array}$ & $\begin{array}{l}2.846^{\mathrm{bc}} \\
(2.37)\end{array}$ & $\begin{array}{l}2.842^{\mathrm{bc}} \\
(2.21)\end{array}$ \\
\hline Greater yam peel & $2.057 \mathrm{c}$ & $\begin{array}{l}2.135^{\mathrm{b}} \\
(3.78)\end{array}$ & $\begin{array}{l}2.348^{a} \\
(14.14)\end{array}$ & $\begin{array}{l}2.314^{\mathrm{a}} \\
(12.51)\end{array}$ & $\begin{array}{l}2.121^{b} \\
(3.11)\end{array}$ & $\begin{array}{l}2.091^{\mathrm{bc}} \\
(1.66)\end{array}$ & $\begin{array}{l}2.103^{b} \\
(2.23)\end{array}$ \\
\hline Beet root peel & $1.026^{\mathrm{ab}}$ & $\begin{array}{l}1.067^{\mathrm{ab}} \\
(3.97)\end{array}$ & $\begin{array}{l}1.188^{a} \\
(15.83)\end{array}$ & $\begin{array}{l}1.170^{\mathrm{a}} \\
(14.07)\end{array}$ & $\begin{array}{l}1.052^{\mathrm{ab}} \\
(2.55)\end{array}$ & $\begin{array}{l}1.049^{\mathrm{ab}} \\
(2.25)\end{array}$ & $\begin{array}{l}1.048^{\mathrm{ab}} \\
(2.09)\end{array}$ \\
\hline \multicolumn{8}{|c|}{ Vegetable processing residues } \\
\hline Ash gourd peel & $3.016^{c}$ & $\begin{array}{l}3.132^{\mathrm{b}} \\
(3.82)\end{array}$ & $\begin{array}{l}3.511^{\mathrm{a}} \\
(16.41)\end{array}$ & $\begin{array}{l}3.426^{\mathrm{a}} \\
(13.58)\end{array}$ & $\begin{array}{l}3.111^{\mathrm{b}} \\
(3.14)\end{array}$ & $\begin{array}{l}3.102^{\mathrm{b}} \\
(2.84)\end{array}$ & $\begin{array}{l}3.080^{c} \\
(2.10)\end{array}$ \\
\hline Pumpkin peel & $2.961^{\mathrm{d}}$ & $\begin{array}{l}3.053^{c} \\
(3.09)\end{array}$ & $\begin{array}{l}3.424^{a} \\
(15.63)\end{array}$ & $\begin{array}{l}3.359^{\mathrm{ab}} \\
(13.42)\end{array}$ & $\begin{array}{l}3.047^{c} \\
(2.90)\end{array}$ & $\begin{array}{l}3.024^{c} \\
(2.11)\end{array}$ & $\begin{array}{l}3.023^{c} \\
(2.10)\end{array}$ \\
\hline $\begin{array}{l}\text { Vegetable banana } \\
\text { peel }\end{array}$ & $2.917 \mathrm{~cd}$ & $\begin{array}{l}3.041^{\mathrm{b}} \\
(4.28)\end{array}$ & $\begin{array}{l}3.387 a \\
(16.14)\end{array}$ & $\begin{array}{l}3.336^{\mathrm{a}} \\
(14.38)\end{array}$ & $\begin{array}{l}3.000^{\mathrm{bc}} \\
(2.86)\end{array}$ & $\begin{array}{l}2.99 \mathrm{c} \\
(2.56)\end{array}$ & $\begin{array}{l}2.987 \mathrm{c} \\
(2.40)\end{array}$ \\
\hline $\begin{array}{l}\text { Mixed vegetable } \\
\text { waste }\end{array}$ & $2.653^{c}$ & $\begin{array}{l}2.769^{\mathrm{b}} \\
(4.37)\end{array}$ & $\begin{array}{l}3.083^{a} \\
(16.22)\end{array}$ & $\begin{array}{l}3.014^{\mathrm{a}} \\
(13.59)\end{array}$ & $\begin{array}{l}2.731^{b} \\
(2.95)\end{array}$ & $\begin{array}{l}2.702^{\mathrm{bc}} \\
(1.85)\end{array}$ & $\begin{array}{l}2.700^{\mathrm{bc}} \\
(1.77)\end{array}$ \\
\hline
\end{tabular}

${ }^{*}$ Aqueous extract from the original biomass; ${ }^{* *}$ figures in parentheses indicate percentage increase from the original; ST: steam; DSA: dilute sulphuric acid; LRT: lime room temperature $\left(30 \pm 1{ }^{\circ} \mathrm{C}\right)$; LHT: lime high temperature $\left(121{ }^{\circ} \mathrm{C}\right)$; Means with different superscripts in each row are significant at $\mathrm{p}<0.05$. 
Table 2a. Percentage removal of total soluble phenolic (TSP) inhibitors from prehydrolysates of root crop processing residues by non-ionic surfactants (Tween 20 and PEG).

\begin{tabular}{|c|c|c|c|c|c|c|c|}
\hline $\begin{array}{l}\text { Detoxification } \\
\text { treatments }\end{array}$ & Native & $\begin{array}{l}\text { ST } \\
45 \text { min. }\end{array}$ & $\begin{array}{l}S T \\
60 \mathrm{~min} .\end{array}$ & $\begin{array}{l}\text { DSA } \\
60 \text { min. }\end{array}$ & $\begin{array}{l}\text { LRT } \\
24 \mathrm{~h}\end{array}$ & $\begin{array}{l}\text { LRT } \\
48 \mathrm{~h}\end{array}$ & $\begin{array}{l}\text { LHT } \\
60 \mathrm{~min} .\end{array}$ \\
\hline \multicolumn{8}{|c|}{ Sweet potato peel } \\
\hline $\mathrm{T} 1$ & $73.54^{b}$ & $74.38^{\mathrm{b}}$ & $78.05^{\mathrm{ab}}$ & $76.25^{b}$ & $73.58^{\mathrm{b}}$ & $70.04^{b}$ & $71.34^{b}$ \\
\hline $\mathrm{T} 2$ & $76.65^{a}$ & $76.69^{a}$ & $78.75^{\mathrm{a}}$ & $77.45^{\mathrm{a}}$ & $76.29 a$ & $72.78^{a}$ & $72.45^{a}$ \\
\hline T3 & $55.35^{\mathrm{d}}$ & $56.56^{\mathrm{cd}}$ & $57.29^{\mathrm{d}}$ & $56.66^{c}$ & $55.93^{\mathrm{d}}$ & $55.11^{\mathrm{c}}$ & $55.15^{c}$ \\
\hline $\mathrm{T} 4$ & $56.45^{c}$ & $57.26^{c}$ & $60.47 \mathrm{c}$ & $57.30^{c}$ & $56.92^{c}$ & $55.90^{c}$ & $56.11^{c}$ \\
\hline \multicolumn{8}{|c|}{ Elephant foot yam peel } \\
\hline $\mathrm{T} 1$ & $74.06^{b}$ & $74.90^{\mathrm{b}}$ & $78.37 a$ & $76.77 \mathrm{a}$ & $74.10^{\mathrm{b}}$ & $70.56^{b}$ & $71.86^{a}$ \\
\hline $\mathrm{T} 2$ & $76.24^{a}$ & $76.28^{a}$ & $78.57^{a}$ & $77.04^{\mathrm{a}}$ & $75.88^{a}$ & $72.37^{a}$ & $72.04^{a}$ \\
\hline T3 & $52.59 \mathrm{~d}$ & $52.99 \mathrm{~d}$ & $54.31^{\mathrm{c}}$ & $53.41^{\mathrm{c}}$ & $52.95^{\mathrm{d}}$ & $51.85^{c}$ & $52.20^{c}$ \\
\hline $\mathrm{T} 4$ & $54.00^{c}$ & $54.11^{\mathrm{c}}$ & $64.49^{b}$ & $54.45^{b}$ & $54.06^{c}$ & $52.35^{c}$ & $53.93^{\mathrm{b}}$ \\
\hline \multicolumn{8}{|l|}{ Tannia peel } \\
\hline $\mathrm{T} 1$ & $74.57^{b}$ & $75.41^{b}$ & $79.08^{a}$ & $77.28^{\mathrm{b}}$ & $74.61^{b}$ & $71.07^{b}$ & $72.37^{a}$ \\
\hline $\mathrm{T} 2$ & $77.13^{a}$ & $77.17^{a}$ & $79.23^{a}$ & $77.93^{a}$ & $76.77^{a}$ & $73.26^{a}$ & $72.93^{a}$ \\
\hline T3 & $53.96^{d}$ & $54.18^{\mathrm{d}}$ & $55.84^{c}$ & $54.49^{d}$ & $53.98^{d}$ & $52.70^{\mathrm{d}}$ & $53.84 \mathrm{bc}$ \\
\hline $\mathrm{T} 4$ & $55.03^{c}$ & $55.22^{c}$ & $62.04^{b}$ & $55.75^{\mathrm{c}}$ & $55.06^{c}$ & $54.45^{c}$ & $54.86^{b}$ \\
\hline \multicolumn{8}{|l|}{ Beet root peel } \\
\hline $\mathrm{T} 1$ & $74.60^{\mathrm{b}}$ & $75.44^{b}$ & $79.11^{\mathrm{a}}$ & $77.31^{\mathrm{a}}$ & $74.64^{b}$ & $71.10^{\mathrm{b}}$ & $72.40^{\mathrm{a}}$ \\
\hline $\mathrm{T} 2$ & $77.20^{\mathrm{a}}$ & $77.24^{\mathrm{a}}$ & $79.30^{a}$ & $78.00^{a}$ & $76.84^{a}$ & $73.33^{a}$ & $73.00^{\mathrm{a}}$ \\
\hline T3 & $49.70^{\mathrm{d}}$ & $50.15^{c}$ & $50.48^{c}$ & $50.41^{b}$ & $49.99 c$ & $49.56^{c}$ & $49.64^{b}$ \\
\hline $\mathrm{T} 4$ & $50.29 c$ & $50.90^{c}$ & $58.90^{b}$ & $51.26^{b}$ & $50.37 \mathrm{c}$ & $49.96^{c}$ & $50.21^{b}$ \\
\hline \multicolumn{8}{|c|}{ Greater yam peel } \\
\hline $\mathrm{T} 1$ & $74.04^{b}$ & $74.88^{b}$ & $78.32^{\mathrm{a}}$ & $76.75^{\mathrm{a}}$ & $74.08^{b}$ & $70.54^{b}$ & $71.84^{a}$ \\
\hline $\mathrm{T} 2$ & $76.22^{\mathrm{a}}$ & $76.26^{a}$ & $78.55^{\mathrm{a}}$ & $77.02^{\mathrm{a}}$ & $75.86^{a}$ & $72.35^{\mathrm{a}}$ & $72.02^{a}$ \\
\hline T3 & $52.57^{d}$ & $52.97^{\mathrm{d}}$ & $54.29^{c}$ & $53.39^{b}$ & $52.93^{\mathrm{d}}$ & $51.83^{c}$ & $52.18^{c}$ \\
\hline $\mathrm{T} 4$ & $53.98^{c}$ & $54.09^{c}$ & $64.47^{b}$ & $54.43^{\mathrm{b}}$ & $54.04^{c}$ & $52.3^{c}$ & $53.91^{b}$ \\
\hline
\end{tabular}

T1: Tween 20 (0.50\%); T2: (0.75\%); T3: Polyethylene glycol (PEG 4000) (0.25\%); T4: PEG (0.5\%); ST: steam; DSA: dilute sulphuric acid; LRT: lime room temperature $\left(30 \pm 1{ }^{\circ} \mathrm{C}\right)$; LHT: lime high temperature $\left(121^{\circ} \mathrm{C}\right)$; Means with different superscripts in each column for each biomass are significant at $\mathrm{p}<0.05$. 
Table 2b. Percentage removal of total soluble phenolic (TSP) inhibitors from prehydrolysates of vegetable processing residues by non-ionic surfactants (Tween 20 and PEG)*.

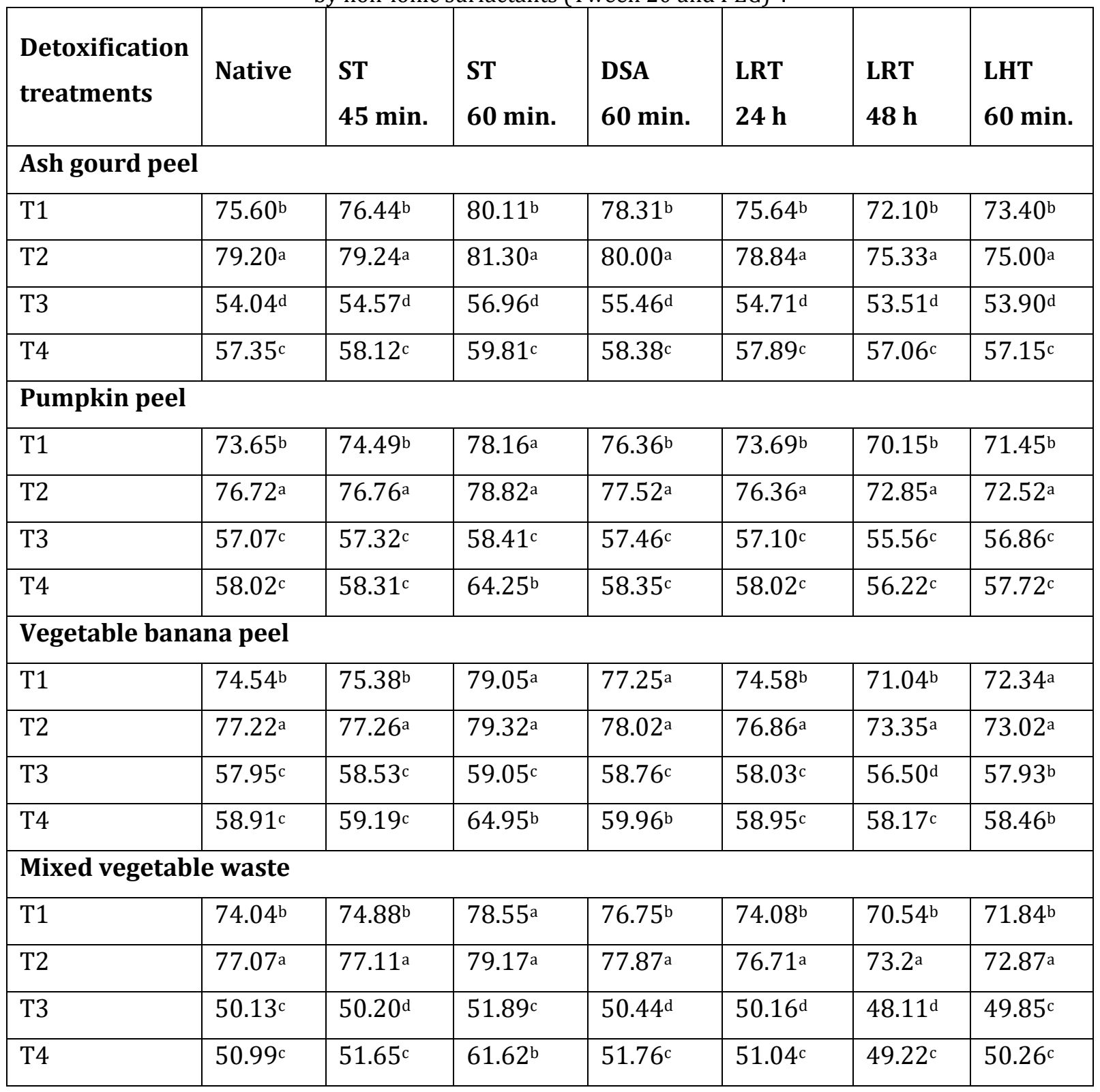

* Footnotes as in Table 2a.

Surfactants with high hydrophilic-lipophilic balance (HLB) such as Tween 20 (HLB 16.7) and PEG 4000 (HLB 18.5) were reported to be more effective in extracting hydrophobic lignin degradation products into the soluble phase [44]. We have found that out of the two surfactants, Tween had a better action in removing phenolics from the prehydrolysates, by possible complex formation with them. This could be advantageous in the case of root and vegetable biomass prehydrolysates rich in fermentable sugars as it could exert a dual effect on the soluble and solid fractions simultaneously.

\subsubsection{Active charcoal}

The effect of active charcoal at two levels such as $1 \%$ and $2 \%$ at room temperature (RT; $30 \pm 1{ }^{\circ} \mathrm{C}$ ) and at $45{ }^{0} \mathrm{C}$ in removing phenols from the prehydrolysates was investigated. When compared to the non-ionic surfactants, the extent of phenol removal was 
Table 3a. Percentage removal of total soluble phenolic (TSP) inhibitors from prehydrolysates of root crop processing residues by active charcoal.

\begin{tabular}{|c|c|c|c|c|c|c|c|}
\hline $\begin{array}{l}\text { Detoxification } \\
\text { treatments }\end{array}$ & Native & $\begin{array}{l}\text { ST } \\
45 \mathrm{~min} .\end{array}$ & $\begin{array}{l}S T \\
60 \mathrm{~min} .\end{array}$ & $\begin{array}{l}\text { DSA } \\
60 \mathrm{~min} .\end{array}$ & $\begin{array}{l}\text { LRT } \\
24 \mathrm{~h}\end{array}$ & $\begin{array}{l}\text { LRT } \\
48 \mathrm{~h}\end{array}$ & $\begin{array}{l}\text { LHT } \\
60 \mathrm{~min} .\end{array}$ \\
\hline \multicolumn{8}{|c|}{ Sweet potato peel } \\
\hline T5 & $31.32^{\mathrm{d}}$ & $34.22^{\mathrm{d}}$ & $41.38^{\mathrm{d}}$ & $36.61^{d}$ & $31.86^{\mathrm{d}}$ & $31.05^{\mathrm{d}}$ & $30.72^{\mathrm{d}}$ \\
\hline T6 & $36.38^{c}$ & $41.90^{c}$ & $47.74^{\mathrm{c}}$ & $43.61^{c}$ & $37.52^{\mathrm{c}}$ & $35.94^{c}$ & $35.20^{c}$ \\
\hline T7 & $41.17^{b}$ & $44.07^{\mathrm{b}}$ & $51.23^{\mathrm{b}}$ & $46.46^{b}$ & $41.71^{b}$ & $40.91^{b}$ & $40.58^{\mathrm{b}}$ \\
\hline T8 & $44.81^{\mathrm{a}}$ & $50.33^{a}$ & $56.17 \mathrm{a}$ & $52.04^{a}$ & $45.95^{\mathrm{a}}$ & $44.37 \mathrm{a}$ & $43.63^{a}$ \\
\hline \multicolumn{8}{|c|}{ Elephant foot yam peel } \\
\hline T5 & $33.75^{d}$ & $36.83^{\mathrm{d}}$ & $44.12^{\mathrm{d}}$ & $39.63^{\mathrm{d}}$ & $34.29 \mathrm{~d}$ & $33.49^{d}$ & $33.21^{\mathrm{d}}$ \\
\hline T6 & $39.21^{c}$ & $43.97^{c}$ & $50.91^{\mathrm{c}}$ & $47.21^{\mathrm{c}}$ & $40.38^{c}$ & $38.76^{c}$ & $38.06^{c}$ \\
\hline T7 & $43.61^{b}$ & $46.68^{b}$ & $53.98^{\mathrm{b}}$ & $49.49^{b}$ & $44.14^{b}$ & $43.35^{b}$ & $43.07 \mathrm{~b}$ \\
\hline T8 & $47.64^{a}$ & $52.40^{\mathrm{a}}$ & $59.34^{\mathrm{a}}$ & $55.64^{\mathrm{a}}$ & 48.81 & $47.20^{\mathrm{a}}$ & $46.49^{a}$ \\
\hline \multicolumn{8}{|l|}{ Tannia peel } \\
\hline T5 & $30.93^{d}$ & $34.03^{\mathrm{d}}$ & $40.33^{\mathrm{d}}$ & $36.22^{\mathrm{d}}$ & $31.53^{\mathrm{d}}$ & $30.92^{d}$ & $30.40^{\mathrm{d}}$ \\
\hline T6 & $36.29 c$ & $40.98^{c}$ & $46.53^{c}$ & $43.15^{c}$ & $37.13^{c}$ & $35.38^{c}$ & $34.84^{c}$ \\
\hline T7 & $40.79^{b}$ & $43.89^{b}$ & $50.18^{b}$ & $46.08^{b}$ & $41.38^{b}$ & $40.77^{b}$ & $40.26^{b}$ \\
\hline T8 & $44.72^{\mathrm{a}}$ & $49.41^{a}$ & $54.96^{\mathrm{a}}$ & $51.58^{a}$ & $45.56^{\mathrm{a}}$ & $43.81^{a}$ & $43.27^{a}$ \\
\hline \multicolumn{8}{|l|}{ Beet root peel } \\
\hline T5 & $34.11^{\mathrm{d}}$ & $36.56^{d}$ & $42.07^{d}$ & $38.45^{\mathrm{d}}$ & $34.21^{\mathrm{d}}$ & $33.93^{\mathrm{d}}$ & $33.03^{\mathrm{d}}$ \\
\hline T6 & $39.96^{c}$ & $42.18^{c}$ & $48.80^{c}$ & $45.28^{c}$ & $40.86^{c}$ & $39.08^{c}$ & $39.04^{c}$ \\
\hline $\mathrm{T} 7$ & $43.97 \mathrm{~b}$ & $46.41^{b}$ & $51.93^{b}$ & $48.30^{b}$ & $44.07 \mathrm{~b}$ & $43.79^{b}$ & $42.88^{b}$ \\
\hline T8 & $48.39 a$ & $50.61^{a}$ & $57.23^{\mathrm{a}}$ & $53.71^{a}$ & $49.29^{a}$ & $47.51^{a}$ & $47.47^{a}$ \\
\hline \multicolumn{8}{|c|}{ Greater yam peel } \\
\hline T5 & $33.73^{\mathrm{d}}$ & $36.81^{\mathrm{d}}$ & $44.10^{\mathrm{d}}$ & $39.61^{d}$ & $34.27 \mathrm{~d}$ & $33.47^{\mathrm{d}}$ & $33.19^{\mathrm{d}}$ \\
\hline T6 & $39.19 c$ & $43.95^{c}$ & $50.89^{c}$ & $47.19^{c}$ & $40.36^{c}$ & $38.74^{c}$ & $38.04^{c}$ \\
\hline $\mathrm{T} 7$ & $43.59^{b}$ & $46.66^{b}$ & $53.96^{b}$ & $49.47^{b}$ & $44.12^{b}$ & $43.33^{b}$ & $43.05^{b}$ \\
\hline T8 & $47.62^{\mathrm{a}}$ & $52.38^{a}$ & $59.32^{\mathrm{a}}$ & $55.62^{a}$ & $48.79^{a}$ & $47.18^{a}$ & $46.47^{a}$ \\
\hline
\end{tabular}

T5: Active charcoal $\left(1.0 \% ; 30 \pm 1{ }^{\circ} \mathrm{C}\right)$; T6: $\left(2.0 \% ; 30 \pm 1{ }^{\circ} \mathrm{C}\right)$; T7: $\left(1.0 \% ; 45^{\circ} \mathrm{C}\right)$; T8: $\left(2.0 \% ; 45^{\circ} \mathrm{C}\right)$; other footnotes as in Table $2 \mathrm{a}$. 
significantly low (Tables 3 a and b) and ranged from 28$51 \%$ in the prehydrolysates treated with active charcoal at RT. Enhancing the temperature to $45{ }^{\circ} \mathrm{C}$ resulted in significantly higher removal of phenolics (38-59\%), which still was much less than that removed by Tween 20. The extent of removal of inhibitors especially phenolics by active charcoal was highly dependent on $\mathrm{pH}$ and $\mathrm{pH} 2.0$ favoured maximum removal [22].

Table 3b. Percentage removal of total soluble phenolic (TSP) inhibitors from prehydrolysates of vegetable processing residues by active charcoal.

\begin{tabular}{|c|c|c|c|c|c|c|c|}
\hline $\begin{array}{l}\text { Detoxification } \\
\text { treatments }\end{array}$ & Native & $\begin{array}{l}\text { ST } \\
45 \text { min. }\end{array}$ & $\begin{array}{l}S T \\
60 \mathrm{~min} .\end{array}$ & $\begin{array}{l}\text { DSA } \\
60 \mathrm{~min} .\end{array}$ & $\begin{array}{l}\text { LRT } \\
24 \mathrm{~h}\end{array}$ & $\begin{array}{l}\text { LRT } \\
48 \mathrm{~h}\end{array}$ & $\begin{array}{l}\text { LHT } \\
60 \mathrm{~min} .\end{array}$ \\
\hline \multicolumn{8}{|l|}{ Ash gourd peel } \\
\hline T5 & $28.51^{d}$ & $31.29^{d}$ & $37.02^{\mathrm{d}}$ & $33.57^{d}$ & $28.93^{d}$ & $28.37^{d}$ & $28.05^{d}$ \\
\hline T6 & $33.12^{c}$ & $36.40^{c}$ & $42.72^{\mathrm{c}}$ & $39.99 c$ & $34.07 \mathrm{c}$ & $32.46^{c}$ & $32.15^{c}$ \\
\hline T7 & $38.37^{b}$ & $41.15^{b}$ & $46.88^{b}$ & $43.42^{b}$ & $38.79^{b}$ & $38.22^{\mathrm{b}}$ & $37.91^{\mathrm{b}}$ \\
\hline T8 & $41.55^{\mathrm{a}}$ & $44.84^{a}$ & $51.15^{\mathrm{a}}$ & $48.42^{\mathrm{a}}$ & $42.50^{\mathrm{a}}$ & $40.89^{a}$ & $40.58^{a}$ \\
\hline \multicolumn{8}{|l|}{ Pumpkin peel } \\
\hline T5 & $29.04^{\mathrm{d}}$ & $32.10^{\mathrm{d}}$ & $37.97^{\mathrm{d}}$ & $34.24^{\mathrm{d}}$ & $29.54^{d}$ & $28.7^{\mathrm{d}}$ & $28.58^{\mathrm{d}}$ \\
\hline T6 & $33.74 c$ & $37.34^{c}$ & $43.81^{\mathrm{c}}$ & $40.79 \mathrm{c}$ & $34.79 \mathrm{c}$ & $33.30^{c}$ & $32.75^{c}$ \\
\hline $\mathrm{T} 7$ & $38.90^{\mathrm{b}}$ & $41.96^{b}$ & $47.82^{b}$ & $44.09^{\mathrm{b}}$ & $39.39^{b}$ & $38.63^{b}$ & $38.43^{b}$ \\
\hline T8 & $42.17 \mathrm{a}$ & $45.77^{a}$ & $52.24^{a}$ & $49.22^{\mathrm{a}}$ & $43.22^{\mathrm{a}}$ & $41.73^{a}$ & $41.18^{\mathrm{a}}$ \\
\hline \multicolumn{8}{|c|}{ Vegetable banana peel } \\
\hline T5 & $29.49 \mathrm{~d}$ & $32.22^{\mathrm{d}}$ & 38.38 & $34.47^{\mathrm{d}}$ & $30.00^{\mathrm{d}}$ & $29.42^{\mathrm{d}}$ & $28.93^{\mathrm{d}}$ \\
\hline T6 & $34.25^{c}$ & $39.13^{c}$ & $44.29^{c}$ & $41.07^{c}$ & $35.33^{c}$ & $33.66^{c}$ & $33.15^{c}$ \\
\hline T7 & $39.34^{b}$ & $42.08^{b}$ & $48.24^{b}$ & $44.33^{\mathrm{b}}$ & $39.86^{b}$ & $39.27^{b}$ & $38.79^{b}$ \\
\hline T8 & $42.69^{a}$ & $47.56^{\mathrm{a}}$ & $52.72^{\mathrm{a}}$ & $49.50^{\mathrm{a}}$ & $43.77^{a}$ & $42.10^{\mathrm{a}}$ & $41.58^{\mathrm{a}}$ \\
\hline \multicolumn{8}{|c|}{ Mixed vegetable waste } \\
\hline T5 & $32.42^{\mathrm{d}}$ & $35.39^{d}$ & $42.16^{d}$ & $38.16^{\mathrm{d}}$ & $32.95^{\mathrm{d}}$ & $32.19^{\mathrm{d}}$ & 31.98 \\
\hline T6 & $37.65^{c}$ & $42.62^{c}$ & $48.65^{c}$ & $45.46^{c}$ & $38.81^{c}$ & $37.26^{c}$ & $36.64^{c}$ \\
\hline T7 & $42.27 \mathrm{~b}$ & $45.25^{b}$ & $52.02^{\mathrm{b}}$ & $48.02^{\mathrm{b}}$ & $42.81^{b}$ & $42.05^{b}$ & $41.83^{b}$ \\
\hline T8 & $46.09^{a}$ & $51.05^{\mathrm{a}}$ & $57.08^{\mathrm{a}}$ & $53.89^{a}$ & $47.24^{\mathrm{a}}$ & $45.70^{a}$ & $45.07^{a}$ \\
\hline
\end{tabular}

Footnotes as in Tables 2a and 3a.

In the present study the effect of various detoxifying agents was to be compared and hence the same $\mathrm{pH}$ of 6.0 was adopted for all the prehydrolysates uniformly, which might have resulted in lower removal in active charcoal treated prehydrolysates. Besides, a uniform contact time of one hour was adopted in the present study for both the concentrations of active charcoal. Parajó et al [23] reported that maximum removal of lignin degradation products from wood hydrolysates occurred at $20 \mathrm{~min}$. and further increase to $90 \mathrm{~min}$. had no significant effect. 
Table 4. Percentage removal of total soluble phenolic (TSP) inhibitors from prehydrolysates by sodium borohydride.

\begin{tabular}{|c|c|c|c|c|c|c|c|}
\hline $\begin{array}{l}\text { Detoxification } \\
\text { treatments }\end{array}$ & Native & $\begin{array}{l}\text { ST } \\
45 \mathrm{~min} .\end{array}$ & $\begin{array}{l}S T \\
60 \mathrm{~min} .\end{array}$ & $\begin{array}{l}\text { DSA } \\
60 \mathrm{~min} .\end{array}$ & $\begin{array}{l}\text { LRT } \\
24 \mathrm{~h}\end{array}$ & $\begin{array}{l}\text { LRT } \\
48 \mathrm{~h}\end{array}$ & $\begin{array}{l}\text { LHT } \\
60 \mathrm{~min} .\end{array}$ \\
\hline \multicolumn{8}{|c|}{ Sweet potato peel } \\
\hline T9 & $42.53^{\mathrm{c}}$ & $43.29 \mathrm{bc}$ & $46.47 \mathrm{a}$ & $44.88^{\mathrm{b}}$ & $42.86^{c}$ & $41.97 \mathrm{c}$ & $40.89 \mathrm{~d}$ \\
\hline $\mathrm{T} 10$ & $43.44^{\mathrm{d}}$ & $45.39^{c}$ & $49.33^{a}$ & $47.43^{b}$ & $44.81^{c}$ & $42.83^{\mathrm{d}}$ & $41.56^{\mathrm{e}}$ \\
\hline \multicolumn{8}{|c|}{ Elephant foot yam peel } \\
\hline T9 & $45.88^{\mathrm{bc}}$ & $46.60^{\mathrm{b}}$ & $49.55^{\mathrm{a}}$ & $48.59^{a}$ & $46.13^{\mathrm{b}}$ & $45.27 \mathrm{c}$ & $44.55^{\mathrm{d}}$ \\
\hline $\mathrm{T} 10$ & $46.82^{\mathrm{e}}$ & $48.85^{c}$ & $52.61^{a}$ & $51.35^{b}$ & $47.05^{\mathrm{d}}$ & $46.19 \mathrm{e}$ & $45.09^{\mathrm{f}}$ \\
\hline \multicolumn{8}{|l|}{ Tannia peel } \\
\hline T9 & $42.01^{\mathrm{d}}$ & $43.06^{c}$ & $45.29^{a}$ & $44.41^{\mathrm{b}}$ & $42.42^{\mathrm{d}}$ & $41.31^{\mathrm{e}}$ & $40.78^{\mathrm{f}}$ \\
\hline T10 & $43.01^{\mathrm{d}}$ & $45.15^{c}$ & $48.08^{\mathrm{a}}$ & $46.93^{\mathrm{b}}$ & $44.35^{c}$ & $42.16^{\mathrm{e}}$ & $41.38^{\mathrm{f}}$ \\
\hline \multicolumn{8}{|l|}{ Beet root peel } \\
\hline T9 & $38.01^{\mathrm{c}}$ & $39.37 \mathrm{bc}$ & $41.23^{\mathrm{a}}$ & $40.16^{b}$ & $38.96^{c}$ & $37.17^{\mathrm{cd}}$ & $36.94^{\mathrm{d}}$ \\
\hline $\mathrm{T} 10$ & $38.10^{\mathrm{d}}$ & $40.31^{c}$ & $44.59^{a}$ & $41.86^{b}$ & $38.96^{d}$ & $38.03^{\mathrm{d}}$ & $37.32^{\mathrm{e}}$ \\
\hline \multicolumn{8}{|c|}{ Greater yam peel } \\
\hline T9 & $45.86^{\mathrm{cd}}$ & $46.58^{c}$ & $49.53^{a}$ & $48.57^{b}$ & $46.11^{c}$ & $45.25^{\mathrm{d}}$ & $44.53^{\mathrm{e}}$ \\
\hline $\mathrm{T} 10$ & $46.80 \mathrm{e}$ & $48.83^{c}$ & $52.59 a$ & $51.33^{b}$ & $47.03^{\mathrm{d}}$ & $46.17 \mathrm{e}$ & $45.07^{f}$ \\
\hline \multicolumn{8}{|l|}{ Ash gourd peel } \\
\hline T9 & $38.72^{\mathrm{d}}$ & $39.60^{c}$ & $43.57^{a}$ & $41.16^{b}$ & $38.93^{\mathrm{d}}$ & $37.91^{\mathrm{e}}$ & $36.69^{f}$ \\
\hline T10 & $39.55^{\mathrm{e}}$ & $41.51^{\mathrm{c}}$ & $45.85^{\mathrm{a}}$ & $43.49^{b}$ & $40.69^{d}$ & $38.68^{f}$ & $37.96^{\mathrm{f}}$ \\
\hline \multicolumn{8}{|l|}{ Pumpkin peel } \\
\hline T9 & $39.44^{c}$ & $40.62^{b}$ & $42.64^{\mathrm{a}}$ & $41.98^{\mathrm{a}}$ & $39.74^{c}$ & $38.89^{\mathrm{cd}}$ & $38.33^{\mathrm{d}}$ \\
\hline T10 & $40.39 \mathrm{e}$ & $42.58^{c}$ & $45.27^{a}$ & $44.45^{b}$ & $41.55^{\mathrm{d}}$ & $39.68^{f}$ & $38.73^{g}$ \\
\hline \multicolumn{8}{|c|}{ Vegetable banana peel } \\
\hline T9 & $40.05^{c}$ & $40.77^{c}$ & $43.10^{\mathrm{a}}$ & $42.27^{b}$ & $39.97^{\mathrm{cd}}$ & $39.31^{\mathrm{d}}$ & $38.81^{\mathrm{e}}$ \\
\hline T10 & $41.01^{\mathrm{d}}$ & $42.74^{c}$ & $45.76^{a}$ & $44.67^{b}$ & $41.97^{\mathrm{cd}}$ & $40.45^{\mathrm{e}}$ & $39.21^{\mathrm{f}}$ \\
\hline \multicolumn{8}{|c|}{ Mixed vegetable waste } \\
\hline T9 & $44.02^{\mathrm{c}}$ & $44.78^{c}$ & $47.35^{\mathrm{a}}$ & $46.79 \mathrm{~b}$ & $43.97 \mathrm{~d}$ & $43.52^{\mathrm{d}}$ & $42.90 \mathrm{e}$ \\
\hline T10 & $44.97 \mathrm{e}$ & $46.95^{c}$ & $50.27^{a}$ & $49.44^{b}$ & $45.98^{d}$ & $44.41^{\mathrm{e}}$ & $43.34^{\mathrm{f}}$ \\
\hline
\end{tabular}

T9: Sodium borohydride $(20 \mathrm{mM})$; T10: $(40 \mathrm{mM})$; means with different superscripts in each row are significant at $\mathrm{p}<0.05$; other footnotes as in Table 2a. 
Temperature also greatly influenced the removal of phenolics from rice straw prehydrolysates by active charcoal and six fold increase was reported at $45{ }^{\circ} \mathrm{C}$ compared to $25{ }^{0} \mathrm{C}$ [22]. We have however observed that irrespective of the type of biomass, only $30-35 \%$ increase in phenolic removal occurred by increasing the temperature to $45{ }^{\circ} \mathrm{C}$. Varying reports are available on the effective concentration of active charcoal and while $1 \%$ was sufficient to eliminate $94 \%$ of phenolics from pretreated sugarcane bagasse [44], 1:40 (w/w) was found to be optimal for rice straw hemicellulose hydrolysate [22]. We have found that $2 \%(\mathrm{w} / \mathrm{v})$ significantly improved the removal of phenolics, although the extent was much less than Tween 20 .

\subsubsection{Sodium borohydride}

The advantages of sodium borohydride $\left(\mathrm{NaBH}_{4}\right)$ such as direct addition at high concentrations to fermentation vessel and activity at mild conditions of temperature and $\mathrm{pH}$ such as $30{ }^{\circ} \mathrm{C}$ and 6.0 were utilized for in situ detoxification of sugarcane bagasse and spruce hydrolysates [24]. Its effect in removing phenolics from the prehydrolysates of root and vegetable processing residues was therefore studied. Approximately $37-50 \%$ phenolics were removed by $20 \mathrm{mM} \mathrm{NaBH}_{4}$, while $\mathrm{Ca}$. 5-8\% additional removal was obtained with $40 \mathrm{mM} \mathrm{NaBH} 4$ in 20 min. contact time. Phenolic removal was more from steam (60 min.) and DSA pretreated hydrolysates, though these levels were much less than Tween 20 (Table 4). Cavka and Jönsson [24] found that $\mathrm{NaBH}_{4}$ (39-47 mM) significantly enhanced the ethanol yield and productivity of sugarcane bagasse and spruce hydrolysates, the exact mechanism remaining unknown.

\subsubsection{Combination treatments}

The combined effect of surfactants, Tween 20 at half the dose $(0.25 \%)$ and PEG at $0.25 \%$ was also studied and it was found that as high as $73-82 \%$ removal of phenolics was possible (Fig. 1 a-i). The synergistic action could also help reduce the level of Tween 20 addition, thereby reducing the overall process costs. Synergistic effect of $\mathrm{NaBH}_{4}(20 \mathrm{mM})$ and active charcoal $(1 \%)$ at room temperature for one hour in removing phenolics was also studied and it was found that detoxification was significantly improved compared to either of the two materials alone (Fig. $1 \mathrm{a}-\mathrm{i}$ vs. Tables 3 and 4). However, these values were also much lower than the reduction brought about by Tween 20 alone or its combination with PEG, indicating the superiority of these treatments over others.

\subsection{Loss of Reducing Sugars}

The presence of high amounts of reducing sugars in the prehydrolysates from root and vegetable processing residues [25-27] necessitate whole slurry saccharification to maximize the fermentable sugar yield. Hence the effect of the detoxification treatments in removing reducing sugars along with the inhibitory phenolics was also quantified. While the detoxification treatment effect was evident, there was not much difference between the various prehydrolysates in the extent of sugar removal by each chemical such as Tween 20, PEG 4000, active charcoal or $\mathrm{NaBH}_{4}$. Hence, the mean value from the native and prehydrolysates were taken for each detoxification chemical in the case of each biomass and represented in Table 5. It was found that irrespective of the type of biomass, the least removal of sugars occurred in Tween 20 treatment (T1) followed by T2. Loss of sugars in PEG treated set was $3.7-4.6 \%$ in $\mathrm{T} 3$ and $5.1-6.0 \%$ in $\mathrm{T} 4$. Maximum loss of reducing sugars was observed in active charcoal (2\%) at $45{ }^{0} \mathrm{C}$ followed by $2 \%$ at room temperature. Nevertheless, Silva et al [45] found that treatment of sugarcane bagasse hydrolysate with active charcoal (1\%) resulted in sugar loss of only $0.47 \%$. Sodium borohydride treatment resulted in $6.0-8.8 \%$ loss of sugars. Although Tween 20+PEG treatment brought about higher removal of phenolics compared to Tween 20 alone, the loss of reducing sugars was significantly higher in the combination treatment. As 1-2\% additional loss of reducing sugars could reduce the ethanol yield also considerably, treatment with Tween 20 alone is considered as the best in removing the toxic phenolic inhibitors from the prehydrolysates of root and vegetable processing residues. 

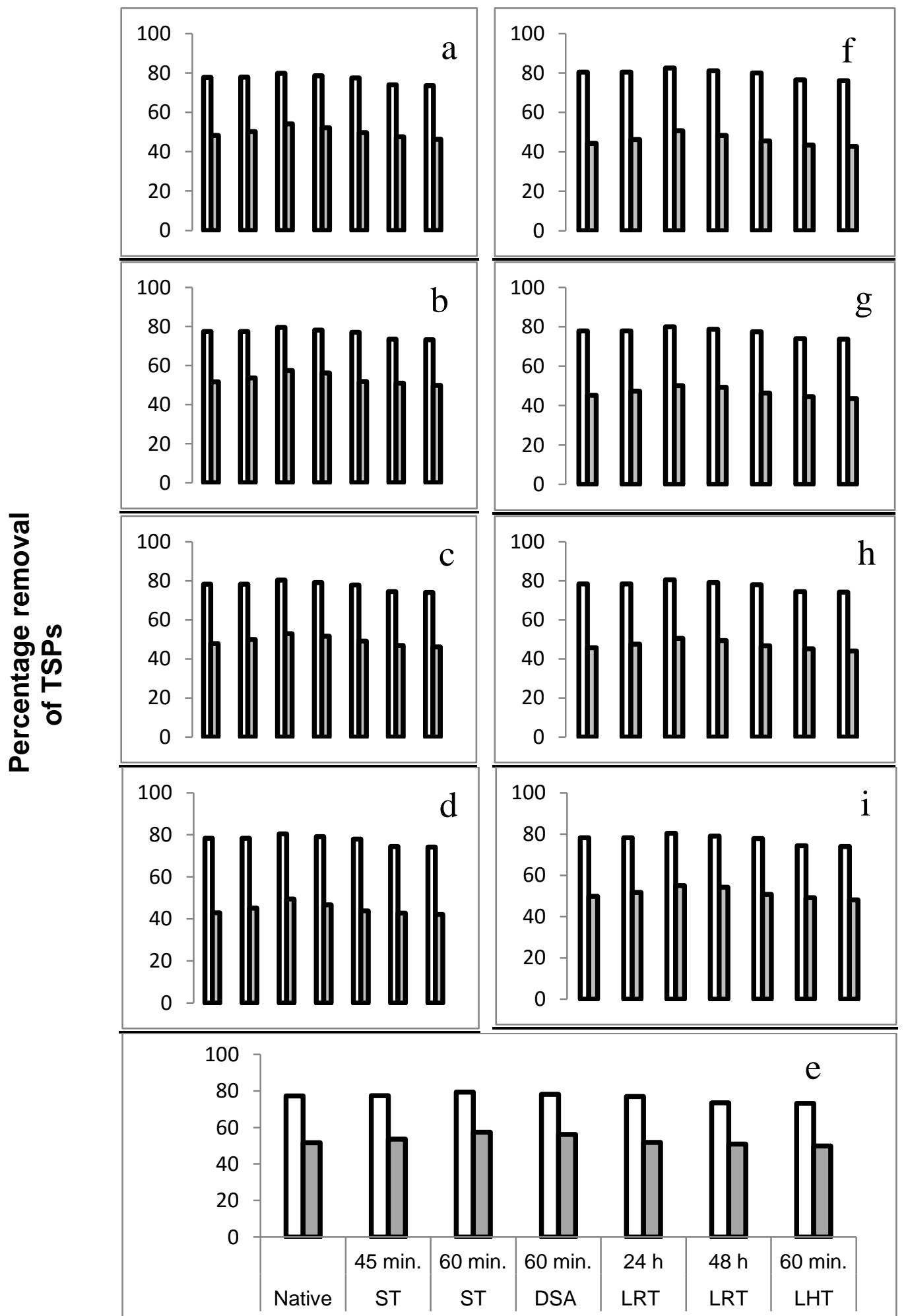

Figure 1. Percentage removal of TSPs from prehydrolysates by combination treatments Bar 1: T11: Tween $20(0.25 \%)+$ PEG (0.25\%); Bar 2: T12: Sodium borohydride (20 mM) + active charcoal (1.0\%), a - e (peels from sweet potato, elephant foot yam, tannia, beet root and greater yam); $\mathrm{f}$ - h (peels from ash gourd, pumpkin and vegetable banana); i- mixed vegetable waste. 
Table 5. Mean percentage reduction in reducing sugars in various detoxification treatments*.

\begin{tabular}{|l|l|l|l|l|l|l|l|l|l|l|l|l|}
\hline \multirow{2}{*}{ Biomass } & \multicolumn{6}{|l}{ Treatments } \\
\cline { 2 - 10 } & T1 & T2 & T3 & T4 & T5 & T6 & T7 & T8 & T9 & T10 & T11 & T12 \\
\hline Sweet potato peel & $2.43^{\mathrm{h}}$ & $3.76^{\mathrm{g}}$ & $3.87^{\mathrm{g}}$ & $5.34^{\mathrm{ef}}$ & $7.27^{\mathrm{d}}$ & $9.21^{\mathrm{b}}$ & $8.26^{\mathrm{c}}$ & $10.47^{\mathrm{a}}$ & $6.33^{\mathrm{e}}$ & $8.15^{\mathrm{c}}$ & $4.62^{\mathrm{f}}$ & $8.21^{\mathrm{c}}$ \\
\hline Elephant foot yam peel & $3.08^{\mathrm{h}}$ & $4.41^{\mathrm{g}}$ & $4.61^{\mathrm{g}}$ & $6.07^{\mathrm{e}}$ & $7.92^{\mathrm{d}}$ & $9.87^{\mathrm{b}}$ & $8.91^{\mathrm{c}}$ & $11.12^{\mathrm{a}}$ & $6.98^{\mathrm{de}}$ & $8.80^{\mathrm{c}}$ & $5.28^{\mathrm{f}}$ & $8.87^{\mathrm{c}}$ \\
\hline Tannia peel & $2.82^{\mathrm{g}}$ & $4.14^{\mathrm{f}}$ & $4.9^{\mathrm{f}}$ & $5.75^{\mathrm{de}}$ & $7.66^{\mathrm{cd}}$ & $9.60^{\mathrm{b}}$ & $8.65^{\mathrm{c}}$ & $10.86^{\mathrm{a}}$ & $6.72^{\mathrm{d}}$ & $8.54^{\mathrm{c}}$ & $5.01^{\mathrm{e}}$ & $8.60^{\mathrm{c}}$ \\
\hline Beetroot peel & $2.9^{\mathrm{i}}$ & $3.61^{\mathrm{gh}}$ & $3.90^{\mathrm{gh}}$ & $5.37^{\mathrm{f}}$ & $7.13^{\mathrm{d}}$ & $9.07^{\mathrm{b}}$ & $8.12^{\mathrm{c}}$ & $10.33^{\mathrm{a}}$ & $6.18^{\mathrm{e}}$ & $8.01^{\mathrm{c}}$ & $4.48^{\mathrm{g}}$ & $8.07^{\mathrm{c}}$ \\
\hline Greater yam peel & $3.07^{\mathrm{h}}$ & $4.39^{\mathrm{g}}$ & $4.59^{\mathrm{g}}$ & $6.06^{\mathrm{ef}}$ & $7.91^{\mathrm{d}}$ & $9.85^{\mathrm{b}}$ & $8.90^{\mathrm{c}}$ & $11.11^{\mathrm{a}}$ & $6.97^{\mathrm{e}}$ & $8.79^{\mathrm{c}}$ & $5.26^{\mathrm{f}}$ & $8.85^{\mathrm{c}}$ \\
\hline Ash gourd peel & $2.75^{\mathrm{h}}$ & $4.07^{\mathrm{g}}$ & $4.25^{\mathrm{g}}$ & $5.72^{\mathrm{f}}$ & $7.59^{\mathrm{d}}$ & $9.53^{\mathrm{b}}$ & $8.58^{\mathrm{c}}$ & $10.79^{\mathrm{a}}$ & $6.64^{\mathrm{e}}$ & $8.47^{\mathrm{c}}$ & $4.79^{\mathrm{g}}$ & $8.53^{\mathrm{c}}$ \\
\hline Pumpkin peel & $2.39^{\mathrm{h}}$ & $3.72^{\mathrm{g}}$ & $3.91^{\mathrm{g}}$ & $5.38^{\mathrm{e}}$ & $7.23^{\mathrm{cd}}$ & $9.18^{\mathrm{b}}$ & $8.22^{\mathrm{c}}$ & $10.43^{\mathrm{a}}$ & $6.29^{\mathrm{d}}$ & $8.11^{\mathrm{c}}$ & $4.59^{\mathrm{f}}$ & $8.18^{\mathrm{c}}$ \\
\hline Vegetable banana peel & $2.08^{\mathrm{h}}$ & $3.41^{\mathrm{g}}$ & $3.67^{\mathrm{g}}$ & $5.14^{\mathrm{ef}}$ & $6.92^{\mathrm{cd}}$ & $8.86^{\mathrm{b}}$ & $7.91^{\mathrm{c}}$ & $10.12^{\mathrm{a}}$ & $5.98^{\mathrm{e}}$ & $7.80^{\mathrm{c}}$ & $4.27^{\mathrm{f}}$ & $7.86^{\mathrm{c}}$ \\
\hline Mixed vegetable waste & $2.63^{\mathrm{h}}$ & $3.95^{\mathrm{fg}}$ & $4.19^{\mathrm{f}}$ & $5.66^{\mathrm{de}}$ & $7.47^{\mathrm{cd}}$ & $9.41^{\mathrm{b}}$ & $8.45^{\mathrm{c}}$ & $10.67^{\mathrm{a}}$ & $6.52^{\mathrm{d}}$ & $8.34^{\mathrm{c}}$ & $4.82^{\mathrm{f}}$ & $8.41^{\mathrm{c}}$ \\
\hline
\end{tabular}

* T1: Tween 20 (0.50\%); T2: (0.75\%); T3: Polyethylene glycol (PEG 4000) (0.25\%); T4: PEG (0.5\%);T5: Active charcoal (1.0\%; $\left.30 \pm 1{ }^{\circ} \mathrm{C}\right)$; T6: $\left(2.0 \% ; 30 \pm 1{ }^{\circ} \mathrm{C}\right)$; T7: $\left(1.0 \% ; 45^{\circ} \mathrm{C}\right)$; T8: $\left(2.0 \% ; 45^{\circ} \mathrm{C}\right)$; T9: Sodium borohydride $(20 \mathrm{mM})$; T10: $(40 \mathrm{mM})$; T11:

Tween $20(0.25 \%)+$ PEG (0.25\%); T12: Sodium borohydride (20 mM) + active charcoal (1.0\%); means with different superscripts in each row are significant at $\mathrm{p}<0.05$.

\section{Conclusion}

Total soluble phenolics (TSPs) ranging from 1-3\% were present in the non-treated aqueous extracts of root and vegetable processing residues. Pretreatments such as steam (60 min; $100{ }^{\circ} \mathrm{C}$ ) and DSA (60 min; 121 ${ }^{0} \mathrm{C}$; $0.102 \mathrm{MPa}$ ) enabled the release of $12.5-16.4 \%$ more phenolics into the soluble fraction, while very low release was observed in the case of lime pretreatment. Maximum effect on the removal of TSPs was exerted by Tween $20(0.25 \%)+$ PEG $4000(0.25 \%)$ for $2 \mathrm{~h}$ at room temperature $\left(30 \pm 1{ }^{\circ} \mathrm{C}\right)$ followed by Tween $20(0.50 \%)$ alone. Loss of reducing sugars was however the least in Tween 20 application. Active charcoal and sodium borohydride removed phenolic inhibitors to a much less extent. Unlike in the case of conventional lignocellulosic hydrolysates, the prehydrolysates from root and vegetable processing residues being rich in fermentable sugars, the whole slurry saccharification and fermentation have to be taken. In this context, Tween 20 with its ability to remove soluble phenolic inhibitors from prehydrolysates and prevent the inhibition of cellulases by the lignin held back in the pretreated residue through effective binding with it, stands out as a dual effect detoxification agent.

\section{Acknowledgements}

The financial support from the Kerala State Council for Science, Technology \& Environment (KSCSTE Grant No. 853/2015/KSCSTE) is gratefully acknowledged. Authors are also thankful to the Director, ICAR- CTCRI for the facilities provided for the study and to Dr. J. Sreekumar, Principal Scientist (Agricultural Statistics) for the help extended in the statistical analyses.

\section{References}

[1] P. Alvira, E. Tomas-Pejo, M. Ballesteros and M. J. Negro, "Pretreatment technologies for an efficient bioethanol production process based on enzymatic hydrolysis: A review," Bioresource Technology, vol. 101, no. 13, pp. 4851-4861, 2010.

[2] A. K. Chandel, R. Rudravaram, M. L. Narasu, V. Rao and P. Ravindra, "Economics and environmental impacts of bioethanol production technologies: an appraisal," Biotechnology and Molecular Biology, vol. 2, no. 1, pp. 14-32, 2007.

[3] B. Yang and C. E. Wyman, "Pretreatment: The key to unlocking low cost cellulosic ethanol," Biofuels, Bioproducts and Biorefining, vol. 2, no. 1, pp. 26-40, 2008. 
[4] N. S. Mosier, C. E. Wyman, B. E. Dale, R. T. Elander, Y. Y. Lee, M. Holtzapple and M. R. Ladisch, "Features of promising technologies for pretreatment of lignocellulosic biomass," Bioresource Technology, vol. 96, no. 6, pp. 673-686, 2005.

[5] C. E. Wyman, B. E. Dale, R. T. Elander, M. Holtzapple, M. R. Ladisch and Y. Y. Lee, "Coordinated development of leading pretreatment technologies," Bioresource Technology, vol. 96, pp. 1959-1966, 2005.

[6] X. Jing, X. Zhang and J. Bao, "Inhibition performance of lignocellulose degradation products on industrial cellulase enzymes during cellulose hydrolysis," Applied Biochemistry and Biotechnology, vol. 159, pp. 696-707, 2009.

[7] H. B. Klinke, A. B. Thomsen and B. K. Ahring, "Inhibition of ethanol-producing yeast and bacteria by degradation products produced during pretreatment of biomass," Applied Microbiology and Biotechnology, vol. 66, pp. 10-26, 2004.

[8] E. Palmqvist and B. Hahn-Hägerdal, "Fermentation of lignocellulosic hydrolysates. II: inhibitors and mechanisms of inhibition," Bioresource Technology, vol. 74, no. 1, pp. 25-33, 2000.

[9] L. J. Jönsson, B. Alriksson and N. O. Nilvebrant, "Bioconversion of lignocellulose: inhibitors and detoxification," Biotechnology for Biofuels, vol. 6, no. 16, 2013.

[10] S. F. Chen, R. A. Mowery, C. J. Scarlata and C. K. Chambliss, "Compositional analysis of watersoluble materials in corn stover," Journal of Agricultural and Food Chemistry, vol. 55, no. 15, pp. 5912-5918, 2007.

[11] E. A. Ximenes, Y. Kim, N. S. Mosier, B. S. Dien and M. R. Ladisch, "Deactivation of cellulases by phenols," Enzyme and Microbial Technology, vol. 48, no. 1, pp. 54-60, 2011.

[12] E. A. Ximenes, Y. Kim, N. S. Mosier, B. S. Dien and M. R. Ladisch, "Inhibition of cellulases by phenols," Enzyme and Microbial Technology, vol. 46, no. 3-4, pp. 170-176, 2010.

[13] A. Tejirian and F. Xu, "Inhibition of enzymatic cellulolysis by phenolic compounds," Enzyme and Microbial Technology, vol. 48, no. 3, pp. 239-247, 2011.

[14] H. Keweloh, G. Weyrauch, H. J. Rehm, "Phenol-induced membrane changes in free and immobilized Escherichia coli," Applied Microbiology and Biotechnology, vol. 33, no. 1, pp. 66-71, 1990.
[15] S. Larsson, A. Quintana-Sáinz, A. Reimann, N. O. Nilvebrant and L. J. Jönsson, "Influence of lignocellulose-derived aromatic compounds on oxygen limited growth and ethanolic fermentation by Saccharomyces cerevisiae," Applied Biochemistry and Biotechnology, vol. 84, pp. 617-632, 2000.

[16] T. A. Clark and K. L. Mackie, "Fermentation inhibitors in wood hydrolysates derived from the softwood Pinus radiata," Journal of Chemical Technology and Biotechnology, vol. 34, no. 2, pp. 101-110. 1984.

[17] L. Olsson and B. Hahn-Hägerdal, "Fermentation of lignocelluloses hydrolysates for ethanol production," Enzyme and Microbial Technology, vol. 18, no. 5, pp. 312-331, 1996.

[18] E. Palmqvist and B. Hahn-Hägerdal, "Fermentation of lignocellulosic hydrolysates. I: inhibition and detoxification," Bioresource Technology, vol. 74, no.1, pp. 17-24, 2000.

[19] W. Parawira and M. Tekere, "Biotechnological strategies to overcome inhibitors in lignocellulose hydrolysates for ethanol production: review," Critical Reviews in Biotechnology, vol. 31, no. 1, pp. 20-31, 2011.

[20] J. Börjesson, R. Peterson and F. Tjerneld, "Enhanced enzymatic conversion of softwood lignocelluloses by poly (ethylene glycol) addition," Enzyme and Microbial Technology, vol. 40, no. 4, pp. 754-762, 2007.

[21] T. Eriksson, J. Börjesson and F. Tjerneld, "Mechanism of surfactant effect in enzymatic hydrolysis of lignocellulose," Enzyme and Microbial Technology, vol. 31, no. 3, pp. 353-364, 2002.

[22] S. I. Mussatto and I. C. Roberto, "Alternatives for detoxification of diluted-acid lignocellulosic hydrolysates for use in fermentative processes: a review," Bioresource Technology, vol. 93, no. 1, pp. 1-10, 2004.

[23] J. C. Parajó, H. Dominguez and J. M. Dominguez, "Charcoal adsorption of wood hydrolysates for improving their fermentability: influence of the operational conditions," Bioresource Technology, vol. 157, no. 2, pp. 179-185, 1996.

[24] A. Cavka and L. J. Jönsson, "Detoxification of lignocellulosic hydrolysates using sodium borohydride," Bioresource Technology, vol. 136, pp. 368-376, 2013.

[25] M. G. Mithra and G. Padmaja, "Compositional profile and ultrastructure of selected root and vegetable processing residues subjected to steam 
and dilute sulphuric acid pretreatment," Current Biotechnology (In press), 2016.

[26] M. G. Mithra and G. Padmaja, "Lime pretreatment associated compositional and ultrastructural changes in selected root and vegetable processing residues," International Journal of Renewable Energy Research (In press), 2016.

[27] M. G. Mithra and G. Padmaja. "Comparative alterations in the compositional profile of selected root and vegetable peels subjected to three pretreatments for enhanced saccharification," Indian Journal of Biotechnology (In press), 2016.

[28] M. P. Divya Nair, G. Padmaja, M. S. Sajeev and J. T. Sheriff, "Bioconversion of cellulo-starch waste from cassava starch industries for ethanol production: Pretreatment techniques and improved enzyme systems," Industrial Biotechnology, vol. 8, no. 5, pp. 300-308, 2012.

[29] V. L. Singleton and A. Rossi, "Colorimetry of total phenolics with phosphomolybdic-phosphotungstic acid reagents," American Journal of Enology and Viticulture, vol. 16, pp. 144-158, 1965.

[30] N. Nelson, "A photometric adaptation of the Somogyi method for determination of glucose," Journal of Biological Chemistry, vol. 153, pp. 375380, 1944.

[31] SAS, SAS/STAT Software Version 9.3, Cary, NC.: SAS Institute Inc., 2010.

[32] M. R. Juntheikki and R. Julkunen-Tiito, "Inhibition of $\beta$-glucosidase and esterase by tannins from Betula, Salix and Pinus species," Journal of Chemical Ecology, vol. 26, no. 5, pp. 1151-1165, 2000.

[33] E. Palmqvist, B. Hahn-Hägerdal, M. Galbe and G. Zacchi, "The effect of water-soluble inhibitors from steam-pretreated willow on enzymatic hydrolysis and ethanol fermentation," Enzyme and Microbial Technology, vol. 19, no. 6, pp. 470-476, 1996.

[34] B. Du, L. N. Sharma, C. Becker, S. F. Chen, R. A. Mowery, G. P. Van Walsum, C.K. Chambliss, "Effect of varying feedstock-pretreatment chemistry combinations on the formation and accumulation of potentially inhibitory degradation products in biomass hydrolysates," Biotechnology and Bioengineering, vol. 107, no. 3, pp. 430-440, 2010.

[35] H. B. Klinke, B. A. Ahring, A. S. Schmidt, A. B. Thomsen, "Characterization of degradation products from alkaline wet oxidation of wheat straw," Bioresource Technology, vol. 82, no. 1, pp. 15-26. 2002.
[36] J. J. Fenske, D. A. Griffin and M. H. Penner, "Comparison of aromatic monomers in lignocellulosic biomass prehydrolysates," Journal of Industrial Microbiology and Biotechnology, vol. 20, no. 6, pp. 364-368, 1998.

[37] Y. Kim, E. A. Ximenes, N. S. Mosier and M. R. Ladisch, "Soluble inhibitors/deactivators of cellulase enzymes from lignocellulosic biomass," Enzyme and Microbial Technology, vol. 48, no. 4-5, pp. 408-415, 2011.

[38] A. Romani, H. A. Ruiz, F. B. Pereira, L. Domingues and J. A. Teixeira, "Effect of hemicelluloses liquid phase on the enzymatic hydrolysis of autohydrolyzed Eucalyptus globulus wood," Biomass Conversion and Biorefinery, vol. 4, no. 2, 2013.

[39] L. J. Jönsson, E. Palmqvist, N. O. Nilvebrant and B. Hahn-Hägerdal, "Detoxification of wood hydrolysates with laccase and peroxidase from the white-rot fungus Trametes versicolor," Applied Microbiology and Biotechnology, vol. 49, no. 6, pp. 691-697, 1998.

[40] A. T. W. M. Hendriks and G. Zeeman, "Pretreatments to enhance the digestibility of lignocellulosic biomass," Bioresource Technology, vol. 100, no. 1, pp. 10-18, 2009.

[41] J. Xu, J. J. Chang, R. R. S. Shivappa and J. C. Burns, "Lime pretreatment of switchgrass at mild temperatures for ethanol production," Bioresource Technology, vol. 101, no. 8, pp. 2900-2903, 2010.

[42] H. J. Heipieper, F. J. Weber, J. Sikkema, H. Kewelo and J. A. M. de Bont, "Mechanism of resistance of whole cells to toxic organic solvents," Trends in Biotechnology, vol. 12, no. 10, pp. 409-415, 1994.

[43] S. Ando, I. Arai, K. Kiyoto and S. Hanai, "Identification of aromatic monomers in steamexploded poplar and their influences on ethanol fermentation by Saccharomyces cerevisiae," Journal of Fermentation Technology, vol. 64, no. 6, pp. 567570, 1986.

[44] M. Kurakake, H. Ooshima, J. Kato, Y. Harano, "Pretreatment of bagasse by non-ionic surfactant for the enzymatic hydrolysis," Bioresource Technology, vol. 49, no. 3, pp. 247-251, 1994.

[45] S. S. Silva, M. G. A. Felipe, M. Vitolo, "Xylitol production by Candida guilliermondii FTI 20037 grown in pretreated sugarcane bagasse hydrolysate," Sustainable Agriculture and Food Energy Industry, pp. 1116-1119, 1998. 